\title{
A NEW UNDERSTANDING OF THE EUROPA ATMOSPHERE AND LIMITS ON GEOPHYSICAL ACTIVITY
}

\author{
D. E. Shemansky ${ }^{1}$, Y. L. Yung ${ }^{2}$, X. Liu ${ }^{1}$, J. YoshiI ${ }^{1}$, C. J. Hansen ${ }^{3}$, A. R. Hendrix ${ }^{4}$, And L. W. Esposito ${ }^{5}$ \\ ${ }^{1}$ Planetary and Space Science Division, Space Environment Technologies, Pasadena, CA 91107, USA; dshemansky@ spacewx.com \\ ${ }^{2}$ Division of Geological and Planetary Sciences, California Institute of Technology, CA 91125, USA \\ ${ }^{3}$ Planetary Science Institute, 109 S. Puerto Drive, Ivins, UT 84738, USA \\ ${ }^{4}$ Planetary Science Institute, Los Angeles, CA 91001, USA \\ ${ }^{5}$ Laboratory for Atmospheric and Space Physics, University of Colorado, CO 80303, USA \\ Received 2014 August 28; accepted 2014 October 31; published 2014 December 2
}

\begin{abstract}
Deep extreme ultraviolet spectrograph exposures of the plasma sheet at the orbit of Europa, obtained in 2001 using the Cassini Ultraviolet Imaging Spectrograph experiment, have been analyzed to determine the state of the gas. The results are in basic agreement with earlier results, in particular with Voyager encounter measurements of electron density and temperature. Mass loading rates and lack of detectable neutrals in the plasma sheet, however, are in conflict with earlier determinations of atmospheric composition and density at Europa. A substantial fraction of the plasma species at the Europa orbit are long-lived sulfur ions originating at Io, with $\sim 25 \%$ derived from Europa. During the outward radial diffusion process to the Europa orbit, heat deposition forces a significant rise in plasma electron temperature and latitudinal size accompanied with conversion to higher order ions, a clear indication that mass loading from Europa is very low. Analysis of far ultraviolet spectra from exposures on Europa leads to the conclusion that earlier reported atmospheric measurements have been misinterpreted. The results in the present work are also in conflict with a report that energetic neutral particles imaged by the Cassini ion and neutral camera experiment originate at the Europa orbit. An interpretation of persistent energetic proton pitch angle distributions near the Europa orbit as an effect of a significant population of neutral gas is also in conflict with the results of the present work. The general conclusion drawn here is that Europa is geophysically far less active than inferred in previous research, with mass loading of the plasma sheet $\leqslant 4.5 \times 10^{25}$ atoms s ${ }^{-1}$ two orders of magnitude below earlier published calculations. Temporal variability in the region joining the Io and Europa orbits, based on the accumulated evidence, is forced by the response of the system to geophysical activity at Io. No evidence for the direct injection of $\mathrm{H}_{2} \mathrm{O}$ into the Europa atmosphere or from Europa into the magnetosphere system, as has been observed at Enceladus in the Saturn system, is obtained in the present investigation.
\end{abstract}

Key words: atomic processes - molecular processes - planetary systems - plasmas

Online-only material: color figures

\section{INTRODUCTION}

The most recent published observations of Europa (Roth et al. 2014) show images, obtained using the Hubble Space Telescope (HST), describing plumes observed over a $7 \mathrm{hr}$ period in 2012 December. This was the only reported occasion of a phenomenon forcing strongly nonuniform atmospheric distribution, described as evidence for a geophysically active body with general similarity to the behavior of Io. Stress during the orbital period was inferred to be responsible. Roth et al. (2014) also reported observations in 1999 October and 2012 November showing atomic oxygen emission from the sunlit face, absent plume features. The general conclusion from the report (Roth et al. 2014) is that Europa is a geophysically active body, emitting water vapor. There has been a general assumption that the Europa atmosphere is composed of $\mathrm{H}_{2} \mathrm{O}$ products (Ip 1996; Ip et al. 1998; Smyth \& Marconi 2006).

The presence of a Europa atmosphere was first reported by Hall et al. (1995) with detection of atomic oxygen through the O I ${ }^{3} P-3 s{ }^{3} S_{o}$ and ${ }^{3} P-3 s{ }^{5} S_{o}$ multiplet transitions at $\sim 1304$ and $\sim 1356 \AA$. The relative brightness of the multiplets, designated here as $\mathcal{I}\left({ }^{3} S\right)$ and $\mathcal{I}\left({ }^{5} S\right)$, is a crucial quantity for the interpretation of how the upper states are excited. Hall et al. (1995) obtained $\mathcal{I}\left({ }^{5} S\right) / \mathcal{I}\left({ }^{3} S\right)$ in the range 1.3-3.2. On the basis of this range of values, it was concluded that the emission was produced by dissociative excitation of $\mathrm{O}_{2}$. The argument that $\mathrm{O}_{2}$ was the dominant component of the Europa atmosphere and the research history leading up to this conclusion was discussed at the time by Hunten (1995). Subsequent observations (Hall et al. 1998) provided measurements at Europa and Ganymede giving the ratio $\mathcal{I}\left({ }^{5} S\right) / \mathcal{I}\left({ }^{3} S\right)$ in the range 1.3-2.2 for Europa and a value of 1.3 at Ganymede. The present work disputes the interpretation of this range of values as an exclusive characteristic of dissociative excitation of $\mathrm{O}_{2}$.

The Cassini Ultraviolet Imaging Spectrograph (UVIS) experiment provided observations of Europa and the surrounding region on 2001 January 6 and 12, reported by Hansen et al. (2005). The analysis presented here is confined to the 2001 day of year (DOY) 012 sequences because the 2001 DOY 006 exposures are contaminated by strong Io torus emission. Although the authors (Hansen et al. 2005) did not dispute the specifics of atmospheric content, this work focused on the presence of atomic oxygen at the top of the atmosphere and disputed the claim (Mauk et al. 2003) that a significant neutral torus was present at the orbit of Europa.

The present work presents the results of a more extended analysis of the UVIS observations at the orbit of Europa during the Cassini 2000/2001 encounter. The new analysis utilizes refined flat-fielding and calibration, allowing the extraction of deep exposures for both extreme ultraviolet (EUV) and far ultraviolet (FUV) UVIS spectrographs, whereas previous work (Hansen et al. 2005) was restricted to the FUV. (The Cassini UVIS experiment contains two spectrographs covering the range $550-1950 \AA$ A. A single exposure produces 64 spectra contiguous 


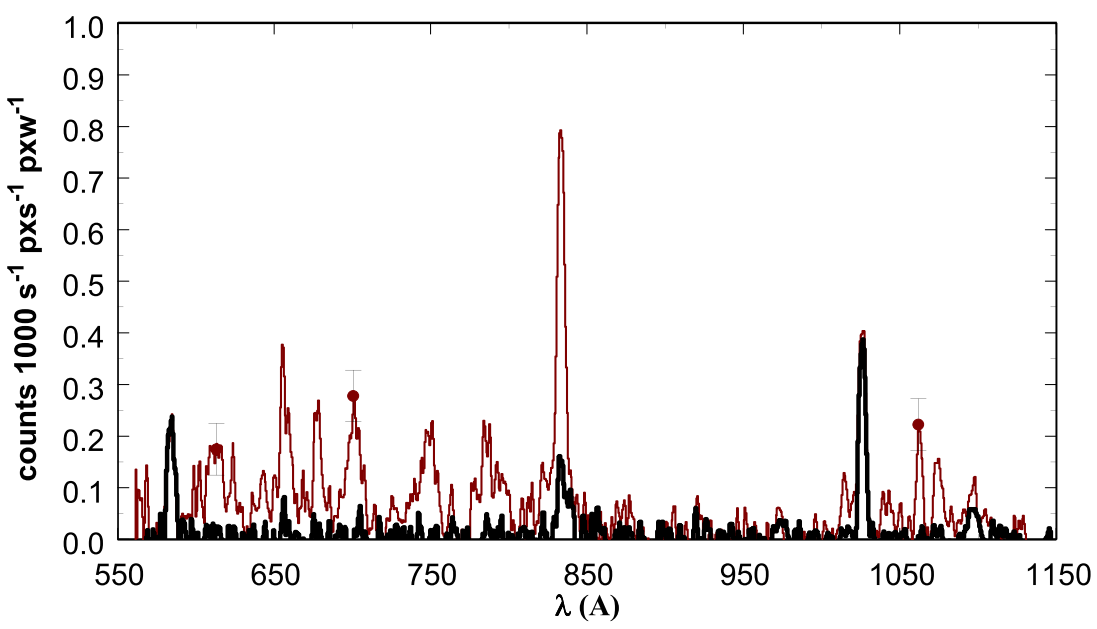

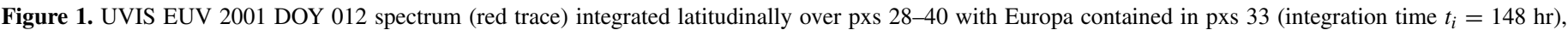

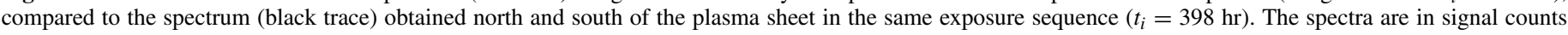

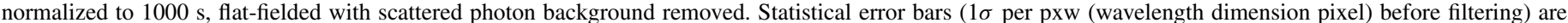

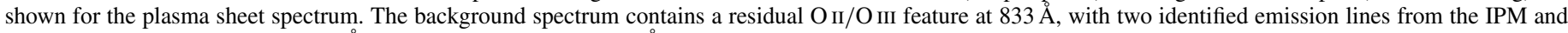

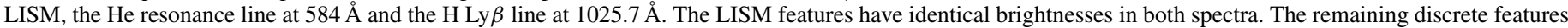
in the plasma sheet spectrum are attributed to oxygen and sulfur ions, primarily outward diffusion products from the Io plasma torus.

(A color version of this figure is available in the online journal.)

in space on $1 \mathrm{mrad}$ centers, as described by Esposito et al. (2004). The establishment of the EUV spectrum has allowed an accurate quantitative determination of the ion species in the plasma sheet at the Europa orbit, setting a strong limit on the mass loading rate from the Europa atmosphere.

\section{CASSINI UVIS EUV SPECTRA OF THE PLASMA SHEET AT EUROPA}

The Cassini UVIS EUV spectrum extracted from the plasma sheet region at Europa is crucial to the determination of the state of the plasma and in setting a limit on the composition of the Europa atmosphere. The geometry of the observations is described in detail by Hansen et al. (2005). The observations were obtained with the Europa image stabilized in a particular spatial pixel (pxs) on the detectors. The spatial dimension of the slit in these observations was aligned parallel to the Jupiter rotational axis, providing latitudinal distribution. Figure 1 shows the integrated flat-fielded signal count spectrum averaged over the latitudinal spatial extent (2.9 Jupiter radii $\left(R_{J}\right)$ ) of the plasma sheet. Figure 1 also shows the averaged spectrum above and below the plasma sheet in the same exposure sequence, containing the foreground/background spectrum. The range to the planet was $\sim 16 \mathrm{Mkm}\left(10^{6} \mathrm{~km}\right)$, in 2001 DOY 012, giving a latitudinal image size of $14 R_{J}$. The dominant feature in the spectrum is the combined $\mathrm{O}_{\text {II }}$ and $\mathrm{O}_{\text {III multiplets near }}$ $833 \AA$, with a Rayleigh brightness of $\sim 1.6 R$. A distinct peak in the plasma emission is not recognizable at the location of Europa, but the distribution shows a FWHM of $\sim 1.5 R_{J}$. The foreground/background spectrum contains two identified discrete emission lines from the interplanetary medium (IPM) and the local interstellar medium (LISM), the He resonance line at $584 \AA$ and the $\mathrm{H} \mathrm{Ly} \beta$ line at $1025.7 \AA$. These features have identical brightnesses in both spectra, as shown in Figure 1. The discrete emission features in the plasma sheet spectrum correspond to those of the emission spectrum from the Io plasma torus, with the exception of the blended transitions in the Europa plasma sheet in the $600-630 \AA$ region found to be spatially mixed with the known emission species. Emission from the minor species $\mathrm{K}$ II and $\mathrm{Cl} I \mathrm{III}$ is possible, but investigation is needed. This region of the spectrum also contains transitions in O II, O III, O IV, and Ov, but the model calculations produce emission levels at least an order of magnitude too weak to account for these features as normal electron impact excitation. It is possible that the emission features are oxygen ion transitions from charge capture reactions (see Shemansky 2003), but this has not been investigated quantitatively. The UVIS Io plasma torus and Europa plasma sheet spectra are compared in Figure 2. The UVIS EUV spectrum of the plasma sheet contains no detectable neutral species emissions. Cassini UVIS observations of the Io torus have been previously reported by Steffl et al. (2004a, 2004b). The latter work examined the local time distribution in the torus and reported distinct temporal variability. The spectrum of the Io torus examined here (Figure 2) is a single exposure at the lower density eastern ansa, to be used as a reference to the plasma at the orbit of Io. The present analysis of the Io spectrum uses flat fielding and absolute calibration that differs from that of Steffl et al. (2004a, 2004b). The latter did not report observations at Europa and therefore are not referenced by Hansen et al. (2005).

\subsection{State of the Plasma Sheet at the Europa Orbit}

Plasma model calculations fitting the EUV plasma sheet spectrum are shown in Figure 3. Models of the LISM emissions are included. The plasma model includes O II, O III, O IV, S II, S III, S IV, and S v emissions calculated for an ambient electron temperature of $T_{e}=250,000 \mathrm{~K}$. The electron temperature used in fitting the observed spectrum at $9.4 R_{J}$ is the same value as that derived in the Voyager 1 Plasma Science Experiment (PLS) measurement of the ambient electrons at this location in the magnetosphere (Sittler \& Strobel 1987). Relative strengths of emission lines at temperatures this high lose sensitivity to the exact value and uncertainty in temperature from emission line relative strength is $\sim \pm 30,000 \mathrm{~K}$. Atomic emission properties in the temperature range below $100,000 \mathrm{~K}$ show much higher sensitivity to the exact temperature value. A model fit to the Io plasma torus east ansa obtained in the Cassini encounter is shown in Figure 4. The states of the plasmas at Io and Europa derived from the model calculations are given in Table 1. 


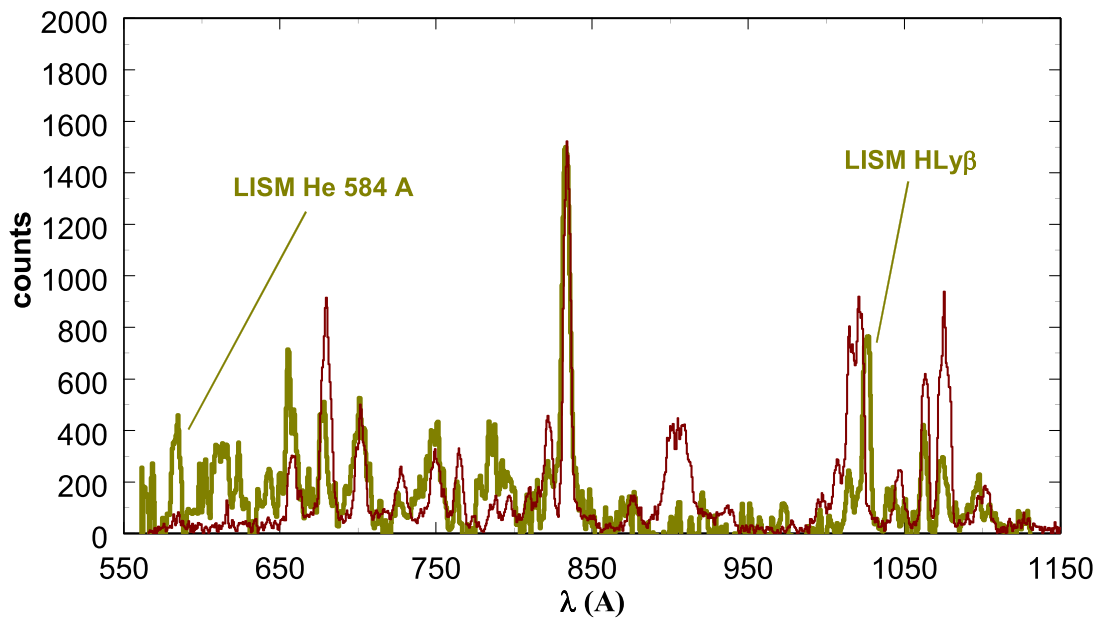

Figure 2. UVIS EUV spectrum of Figure 1 (brown trace) compared to UVIS spectrum of the Io plasma torus eastern ansa (red trace). The spectra are normalized at the O II and O III multiplets near $833 \AA$. The LISM neutral lines appear in the Europa spectrum and not in the Io torus spectrum because of relative brightness. The electron temperature difference between the plasma volumes affect the emission line intensity distributions.

(A color version of this figure is available in the online journal.)

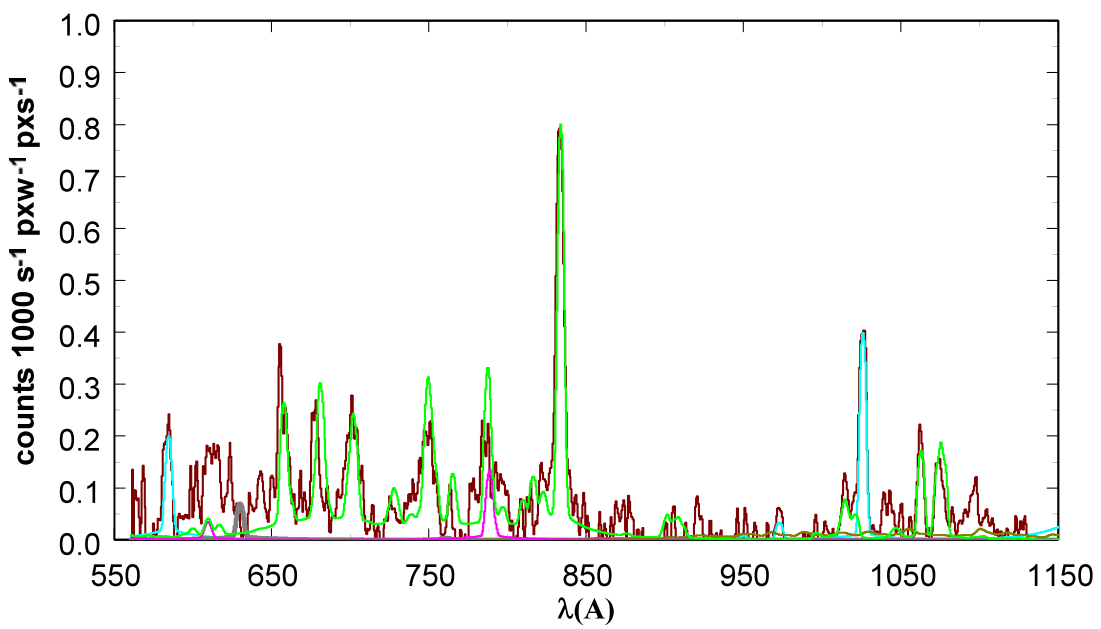

Figure 3. UVIS EUV spectrum of Figure 1 compared to a plasma model calculation including the species O II, O III, O IV, S II, S II, S IV, S v, for an electron temperature $T_{e}=250,000 \mathrm{~K}$. The reduced data is the red trace, the total model is the light green trace, the O IV component is shown in light red, and the LISM emission model is in light blue.

(A color version of this figure is available in the online journal.)

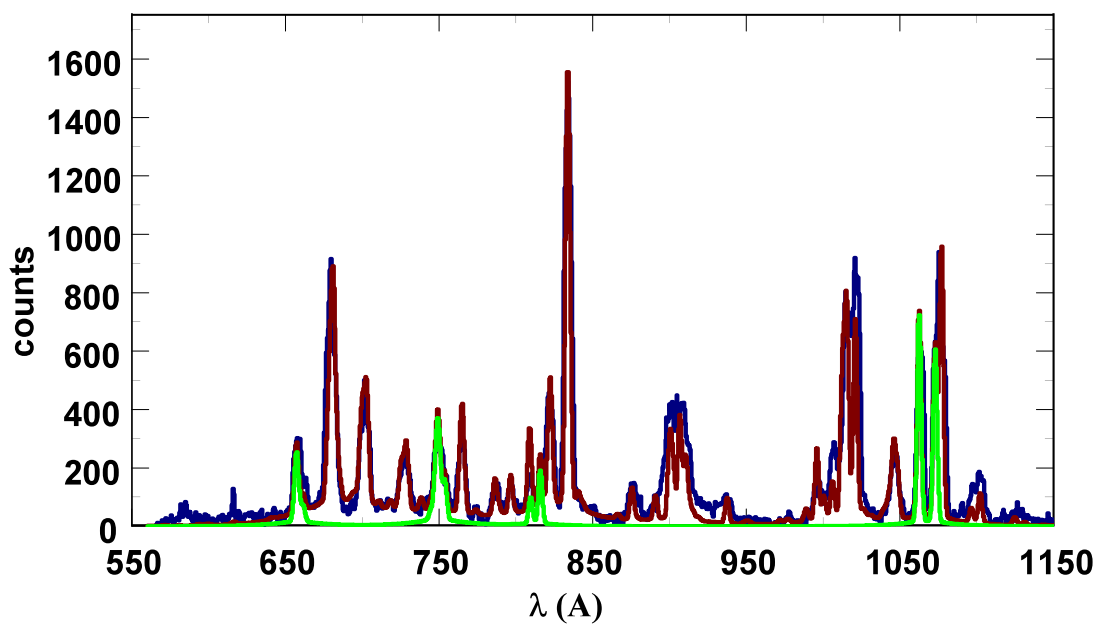

Figure 4. UVIS spectrum of the Io plasma torus eastern ansa (blue trace) compared to a plasma model calculation including the species O II, O III, S II, S III, S IV, S v, for an electron temperature $T_{e}=75,000 \mathrm{~K}$. The total model is the red trace, the $\mathrm{S}$ IV component is shown in light green.

(A color version of this figure is available in the online journal.) 
Table 1

Derived Plasma Properties

\begin{tabular}{|c|c|c|c|c|}
\hline $\begin{array}{l}\text { Radial Location }{ }^{\mathrm{a}} \\
\left(R_{J}\right)\end{array}$ & $5.9^{\mathrm{b}}$ & $5.9^{c}$ & $9.4^{\mathrm{d}}$ & $9.4^{\mathrm{e}}$ \\
\hline$T_{e}(1000 \mathrm{~K})$ & 60. & 75. & $\ldots$ & 250. \\
\hline$\left[\mathrm{OI}_{\mathrm{I}} \mathrm{cm}^{-3}\right.$ & $29 .^{f}$ & $\ldots$ & $\ldots$ & $\ldots$ \\
\hline$[\mathrm{O}$ II $] \mathrm{cm}^{-3}$ & 936. & 118. & 44. & 8.2 \\
\hline$[\mathrm{O} \mathrm{III}] \mathrm{cm}^{-3}$ & 33.8 & 148. & 22. & 13.4 \\
\hline$[\mathrm{O}$ IV $] \mathrm{cm}^{-3}$ & 0.074 & $\leqslant 3$ & $\ldots$ & 4.3 \\
\hline$[\mathrm{S} \mathrm{I}] \mathrm{cm}^{-3}$ & 3.7 & $\ldots$ & $\ldots$ & $\ldots$ \\
\hline$[\mathrm{S}$ II $] \mathrm{cm}^{-3}$ & 287. & 70 . & 6.4 & 1.2 \\
\hline$[\mathrm{S} \mathrm{III}] \mathrm{cm}^{-3}$ & 409 & 355. & 8.1 & 10.7 \\
\hline$\left[\mathrm{S}_{\mathrm{IV}}\right] \mathrm{cm}^{-3}$ & 46.7 & 71. & 5.9 & 7.6 \\
\hline$[\mathrm{S} \mathrm{v}] \mathrm{cm}^{-3}$ & 0.130 & 2.7 & $\ldots$ & 0.75 \\
\hline$\Sigma[\mathrm{Ni}] \mathrm{cm}^{-3}$ & 1745. & 765. & 87.2 & 46.1 \\
\hline$[\mathrm{e}] \mathrm{cm}^{-3}$ & 2250 . & 1418. & 130. & 96. \\
\hline$\Sigma \mathrm{Ni} X \mathrm{~V}^{\mathrm{g}}$ & 60.2 & 26.4 & $\ldots$ & 6.94 \\
\hline$\Sigma[\mathrm{Oi}] /[\mathrm{Si}]$ & 1.34 & 0.53 & 3. & 1.28 \\
\hline $\operatorname{Erad}^{\mathrm{h}}$ & 392. & 565. & $\ldots$ & 7.3 \\
\hline Ecoul $^{\mathrm{i}}$ & 399. & $567 \mathrm{j}^{\mathrm{j}}$ & $\ldots$ & 6.3 \\
\hline
\end{tabular}

Notes.

${ }^{\text {a }}$ Relative to Jupiter center.

b Center of Io hot torus plasma; Voyager 1 encounter 1979 March (Shemansky 1988).

c Center of Io hot torus plasma; Cassini 2001 January.

d Europa location, Voyager PLS 1979 March (Bagenal 1994).

e Europa location, Cassini UVIS 2001 January.

f Theoretical model fit to observation (Shemansky 1988).

$\mathrm{g}$ Total ion content in toroid volume $\left(\times 10^{33}\right)$.

${ }^{\mathrm{h}}$ Mean volumetric radiative cooling rate $10^{-15} \mathrm{erg} \mathrm{cm}^{-3} \mathrm{~s}^{-1}$.

${ }^{\mathrm{i}}$ Coulomb heating of ambient electrons $10^{-15} \mathrm{erg} \mathrm{cm}^{-3} \mathrm{~s}^{-1}$.

j Requires [eh] > Voyager results (Section 2.1.1).

This table includes results from Voyager 1 encounter in 1979, showing that the plasma at Io is a variable phenomenon. Significant variability at Io on a timescale of two weeks in the Cassini encounter is shown by Steffl et al. (2004b). Ion composition from the Voyager PLS (Bagenal 1994) is listed although ambiguous; the ion spectrum produced by this instrument was on an energy per charge scale, so that for example $\mathrm{O}$ II and S III were coincident in the signal output spectrum and therefore not uniquely extractable. The Voyager PLS experiment provides valuable insight into the electron density and temperature distribution between 5.9 and $9.4 R_{J}$ (Sittler \& Strobel 1987), discussed in Section 2.1.1. Table 1 includes the calculated mean volumetric radiative cooling rates, which constitute $\sim 80 \%$ of the energy expended to maintain the plasma in steady state (Shemansky 1988). The quantitative significance of the energy rates is discussed in Section 2.1.1. Plasma energy partitioning is discussed by Sittler \& Strobel (1987) and Shemansky (1988). Sulfur ions, originating from Io, at the orbit of Europa are an expected component in the plasma (Shemansky 1980, 1988; Shemansky \& Smith 1981). Evidently the plasma at the Europa orbit is dominated by ions originating at the Io plasma torus. The outward diffusion of ions from 5.9 $R_{J}$ is characterized by substantially increased ambient electron temperatures (Section 2.1.1) accompanied by the conversion to higher order ions. The ratios of ion species densities at $5.9 R_{J}$ over those at $9.4 R_{J}$, shown in Table 2 , based on the present results decrease significantly with increasing positive charge, indicating overall that conversion to higher order ions occurs more rapidly than other loss processes. The mean density of S II at Europa is a factor of 58 below the value at $5.9 R_{J}$, while $\mathrm{S} \mathrm{V}$ at $9.4 R_{J}$ is only a factor of 3.6 less dense than $\mathrm{S} v$ at
Table 2

Radial Ion Density Progression

\begin{tabular}{|c|c|c|c|c|c|c|c|}
\hline Species & O II & O III & O IV & $\mathrm{S}_{\text {II }}$ & S III & S IV & $\mathrm{S} \mathrm{v}$ \\
\hline$r_{i}^{\mathrm{a}}$ & 14.4 & 11.0 & $\leqslant 0.7$ & 58. & 33. & 9.3 & 3.6 \\
\hline
\end{tabular}

Note. ${ }^{\text {a }}$ Number density ratio Cassini UVIS $2001 r_{i}=N_{i}\left(5.9 R_{J}\right) / N_{i}\left(9.4 R_{J}\right)$.

$5.9 R_{J}$. These facts are indicative of low mass loading rates at Europa. The low density of the plasma at $9.4 R_{J}$ indicates that electron impact is the main controlling factor in volumetric rate processes. It is probable that the plasma at $9.4 R_{J}$ is never in statistical equilibrium. The Io plasma torus is known to be time variable on a scale of months, but the slow diffusion rate of ambient ions outward will filter the magnitude of the variation. The lifetime of S IV against electron impact conversion to $\mathrm{S} v$ at $9.4 R_{J}$, for example, is 120 days.

\subsubsection{Energy Budget}

The radiative cooling rates account for $\sim 80 \%$ of the energy required to maintain the plasma (Shemansky 1988; Yoshii 2011). Table 1 includes calculated radiative cooling rates (see Shemansky 1988). The calculated energy deposition rates shown in the table occur through Coulomb electron-electron and proton-electron interaction of the heterogeneous inwardly diffusing hot plasma with the ambient electrons. The results showing the state of the plasma at Voyager 1 encounter are model calculations fitting the Voyager ultraviolet system (UVS) spectrum. For this reason, neutral atom densities are model projections and not measured in the data. The ion densities and electron temperatures are derived from the spectral data; the electron density and temperature from this analysis agree with the in situ results from the Voyager PLS experiment (Sittler \& Strobel 1987). The inbound electron temperature and densities along the spacecraft $(\mathrm{S} / \mathrm{C})$ trajectory extracted from Sittler \& Strobel (1987) but converted to an $R_{J}$ scale from $\mathrm{L}$ shell are given here in Figure 5. The important properties of Figure 5 are the agreement with the UVS results in Table 1 at the Io torus, and the distribution of the ambient electron temperatures and densities between 5.9 and $9.4 R_{J}$. From the Io torus outward, the electron temperature rises and the electron density declines monotonically to $7.4 R_{J}$, where the temperature rises and the density decreases sharply to $8.4 R_{J}$. Following a region of lower temperature to $8.8 R_{J}$, the temperature rises sharply again coincident with a decrease in density extending to $10 . R_{J}$. These characteristics are interpreted here as decreases in mass loading moving outward, allowing the heterogeneous plasma environment to heat the ambient plasma diffusing outward from the Io torus. Comparing the state of the Io torus plasma from the spectral data at Voyager encounter in 1979 (Column 2) and the result from Cassini UVIS in 2001 (Column 3) it is evident that this region is subject to significant variability. The Io torus in 1979 was colder and more dense than in 2001. Radiative cooling in 1979 was lower than in 2001 because of lower temperature. Column 4 of Table 1 shows the analysis of the Voyager PLS ion partitioning (Bagenal 1994) measurement. These results are less reliable because the PLS experiment measured the mass per charge spectrum, so that $\mathrm{O}$ II and S III, for example, are coincident. Nevertheless the Voyager results in total mass ratio comparing 5.9 and $9.4 R_{J}$, are similar to the Cassini UVIS results in 2001. Column 5 of Table 1 shows the Cassini UVIS result at $9.4 R_{J}$, where compared to $5.9 R_{J}$ the temperature is very much higher, but because of low density, radiative cooling 

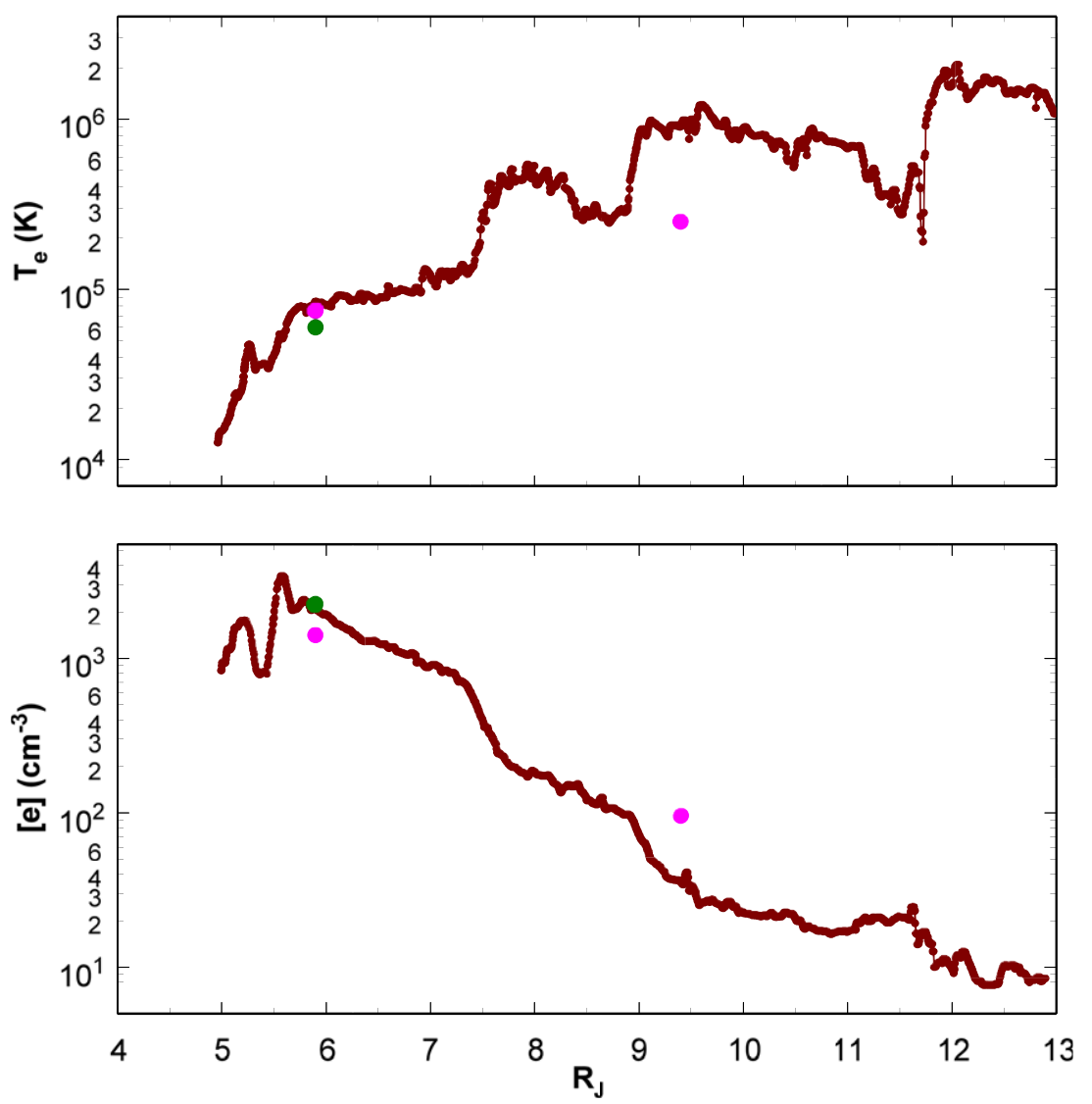

Figure 5. Ambient electron temperatures and densities measured with the PLS experiment on the inbound leg of the Voyager 1 spacecraft in 1979 at Jupiter encounter (from Sittler \& Strobel 1987) transformed to an $R_{J}$ scale, compared to Voyager (green dots) and Cassini (magenta dots) spectrographic plasma emission analysis. There is a general compatibility of PLS with the spectral measurements of the Voyager UVS experiment as well as with the results from the Cassini UVIS experiment in the 2001 encounter.

(A color version of this figure is available in the online journal.)

is reduced by a factor of 54 . The last row of Table 1 shows the calculated energy deposition into the ambient electrons by Coulomb energy transfer (Shemansky 1988; Yoshii 2011). The heterogeneous plasma quantities for the Voyager results at the Io torus (Column 2, Table 1) are from the PLS analysis of Sittler \& Strobel (1987). The calculations for the Io torus in 2001 (Column 3, Table 1), however, require that the hot heterogeneous plasma density in 1979 be increased by a factor of 2.4 in order to approximate the energy deposition rate required to maintain the plasma. For the Cassini UVIS result at $9.4 R_{J}$, the deposition rate calculated using the heterogeneous plasma density from Voyager (Sittler \& Strobel 1987) provides sufficient energy to maintain the observed ambient plasma radiative loss. The results are consistent with the measured conditions in the magnetosphere at Voyager 1 encounter, with, however, a perturbation involving the flow of energy through the Io plasma torus at the time of the Cassini UVIS observations in 2001 as noted above. The results are quantitatively aligned with the argument that the heterogeneous inwardly diffusing magnetospheric plasma is the primary energy source for the maintenance of the Io plasma torus, and that Europa plays a geophysically passive role in the activity in the 5-10 $R_{J}$ region. The energy available for the development of the plasma sheet at $9.4 R_{J}$ is vastly larger than the energy loss rate determined here from observation. The energy required to maintain the Io plasma torus at Voyager 1 encounter, is 54 times greater than the energy required for the plasma sheet at Europa (Table 1), while the primary energy pool for these two regions at the time of Voyager differed by only a factor of less than two. The energy is available at Europa for a plasma on the scale of the Io torus, but Europa does not provide measurable mass loading.

\section{EMISSION PROPERTIES OF THE EUROPA ATMOSPHERE AND OXYGEN RATE PROCESSES}

The UVIS FUV exposures contain the only neutral gas emissions detected in the $9.4 R_{J}$ region from this experiment. Figure 6 shows the 2001 DOY 012 spectrum of the $\mathrm{O}_{1}{ }^{3} P-3 s^{3} S_{o}$ and ${ }^{3} P-3 s{ }^{5} S_{o}$ emissions at and adjacent to the position of Europa on the instrument detector. The solar phase, $\phi=116^{\circ}$, and consequently solar reflection features are not detected in the spectrum. The exposures on 2001 DOY 006 are similar but contaminated with Io torus emission (Hansen et al. 2005). Figure 7 shows plots of the distribution of the primary emission features in Figure 6 as a function of latitudinal distance from Europa (see Hansen et al. 2005). The limits in species abundance is derived from these data (Table 5). The $\mathrm{HI}$ emission is not a measurable plasma sheet source (Figure 1) or a Europa atmosphere source in Figure 7 where the latitudinal distribution of H Ly $\alpha$ emission shows no structure at the location of Europa. Atomic hydrogen emission is thus not found in the plasma sheet in the EUV or as an atmospheric emission in the FUV. The image of Europa falls in pxs 32. The pxs 32 spectrum contains an indication of weak sulfur ion emission from Europa, but these features will not be discussed here. The spectra in pxs 31, 32, and 33, with exposure times of $11 \mathrm{hr}$, are shown along with 


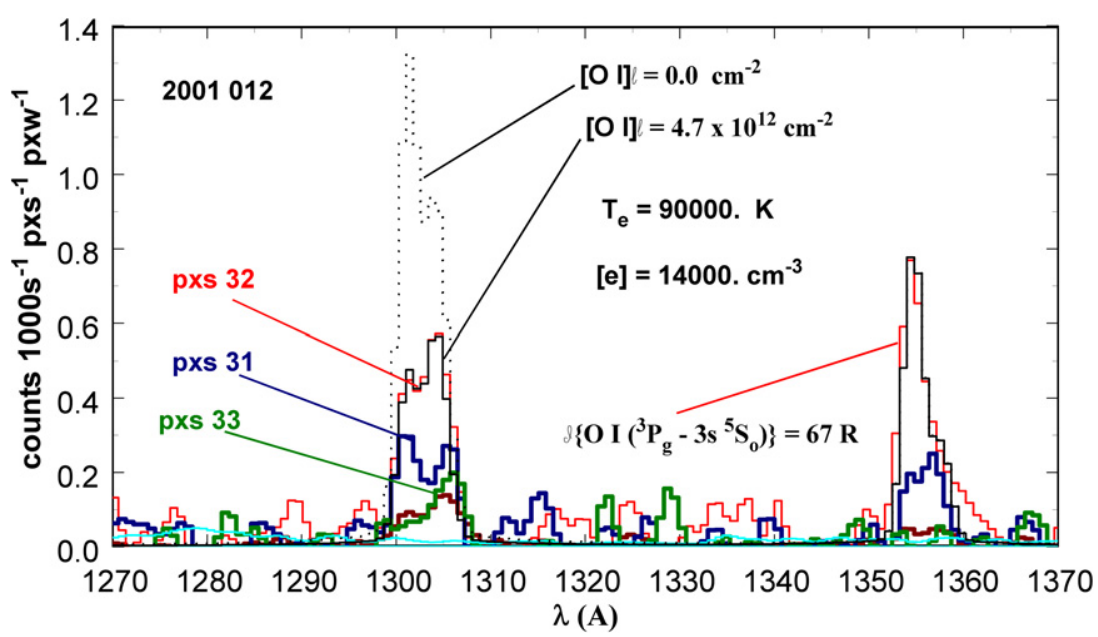

Figure 6. UVIS accumulated FUV spectra (2001 DOY 012) at the Europa ansa, in the 1270.-1370. Å region, compared to model simulations, showing the O I ${ }^{3} P-3 s^{3} S_{o}$ and ${ }^{3} P-3 s^{5} S_{o}$ emission multiplets. Spectra in pxs 31 (blue trace), 32 (light red trace), and 33 (green trace) are shown, with Europa contained in pxs 32 . Integration time is $11 \mathrm{hr}$. The solid black trace is a model calculation with solar and electron forcing, fitting the spectrum in pxs 32 with an opacity abundance of $4.7 \times 10^{12} \mathrm{~cm}^{-2}$. The optically thin model is shown as a dotted black trace. The model calculation shown fitting both O I multiplets requires an electron temperature of $T_{e}=90,000 \mathrm{~K}$. The electron density in the model is [ec] $=14000 \mathrm{~cm}^{-3}$, and the gas temperature $T_{g}=1000 \mathrm{~K}$. The brightness of the O I ${ }^{3} P-3 s^{5} S_{o}$ forbidden multiplet is $67 R$ as indicated on the plot. An upper limit non-LTE model calculation for the excitation of $\mathrm{H}_{2}$ is shown as a light blue trace on the plot.

(A color version of this figure is available in the online journal.)

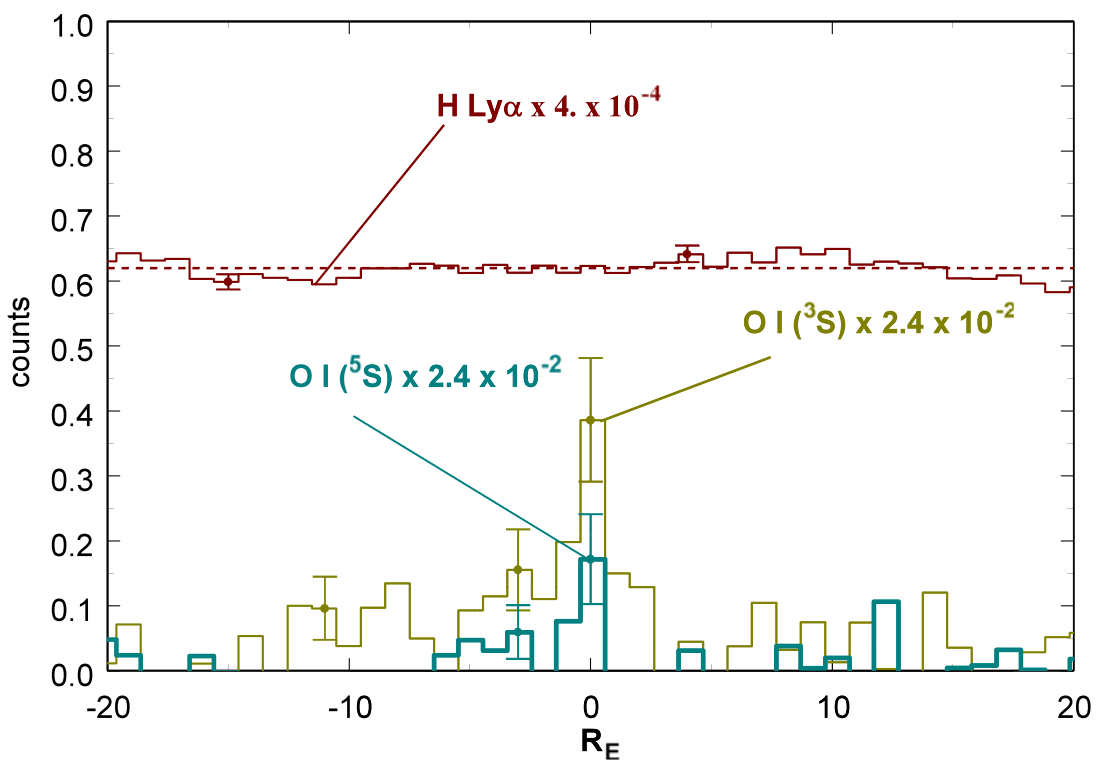

Figure 7. Latitudinal distribution of the $\mathrm{H}$ I and $\mathrm{O}$ I emission features in Figure 6 in distance (Europa radii $R_{E}$ ) from Europa, as identified on the plot. The IPM/LISM emission in $\mathrm{H} \mathrm{Ly} \alpha$ is a spatially uniform foreground/background source competing with the emission from the Europa atmosphere and plasma sheet. There is no evidence for Europa sourced H I emission in these data. Statistical $1 \sigma$ error bars are shown on the plot. As discussed in the text, O I emission features are recognized in only two pixels at Europa.

(A color version of this figure is available in the online journal.)

a modeled statistically equilibrated spectrum of simultaneous electron and solar photon excited $\mathrm{O}$ I, fitting the pxs 32 spectrum. The OI electronic model architecture contains 54 multiplet states (details will not be given here, see Section 3.1). The O I model calculations use the combined forcing of the electron environment and a high resolution solar flux model (Killen et al. 2009). The solid angle of the Europa image on the UVIS detector is 50 times smaller than the pixel size, so it acts as a point source for the spectrograph, giving a spectral resolution of about $2 \AA$. This is sufficient resolution to show the optical thickness in the ${ }^{3} P-3 s{ }^{3} S_{o}$ multiplet transitions. The ${ }^{3} P-3 s{ }^{3} S_{o}$ feature in Figure 6 has evident opacity, and an accurate fit to the multiplet feature has been obtained for a line-of-sight (los) abundance of $4.7 \times 10^{12} \mathrm{~cm}^{-2}$. The Figure 6 graph shows the optically thin spectrum calculated for the same particle density as the optically thick case but with abundance $\mathrm{O}_{\mathrm{I}} \ell=0.0, \ell=0.0$. $\mathrm{O}_{\mathrm{I}}$ $\left({ }^{3} P-3 s{ }^{3} S_{o}\right) /\left({ }^{3} P-3 s{ }^{5} S_{o}\right)$ multiplet pair is accurately modeled at an electron temperature of $T_{e}=90,000 \mathrm{~K}$, as shown in Figure 6 . The presence of $\mathrm{O}_{2}$ is not required to explain the $\mathrm{O}$ I emission in Figure 6 . The spectrum in pxs 31 shows a much smaller opacity and the intensity ratio $\mathcal{I}\left({ }^{5} S\right) / \mathcal{I}\left({ }^{3} S\right)$ is commensurately lower, visibly evident in Figure 6. The spectrum in pxs 33 shows no evidence of electron excited $\mathrm{O}$ I. Comparison of these results with previous observations provides useful additional insight. Table 3 shows the compiled observational results on the Europa atmosphere, showing seven results from separate exposures. The 
Table 3

Europa Atmosphere Observations

\begin{tabular}{|c|c|c|c|c|c|c|c|c|}
\hline $\operatorname{Expt}^{\mathrm{a}}$ & Date & $\mathcal{I}\left({ }^{3} S\right)^{\mathrm{b}}$ & $\mathcal{I}\left({ }^{5} S\right)^{\mathrm{c}}$ & $\mathcal{I}(\operatorname{Ly} \alpha)^{\mathrm{d}}$ & {$[\mathrm{Or}] \ell^{\mathrm{e}}$} & {$\left[\mathrm{O}_{2}\right] \ell^{\mathrm{f}}$} & {$[\mathrm{O} \mathrm{II}] \ell$} & $\mathcal{I}\left({ }^{5} S\right) / \mathcal{I}\left({ }^{3} S\right)$ \\
\hline$\overline{\text { UVIS }^{g}}$ & $2001012^{h}$ & $71 . \pm 6 .^{i}$ & $67 . \pm 6$ & $\cdots$ & $4.7^{\mathrm{j}}$ & $\cdots$ & $\leqslant 0.31$ & 0.94 \\
\hline$H S T^{\mathrm{k}}$ & 1994153 & 29. $\pm 6 .{ }^{l}$ & 64. \pm 6 & $\ldots$ & $\ldots$ & $(240 .-1200 .)^{\mathrm{m}}$ & $\ldots$ & 2.2 \\
\hline$H S T^{\mathrm{n}}$ & 1996203 & $30 . \pm 3.2$ & $64 . \pm 4.6$ & $\ldots$ & $\ldots$ & (350.-1100.) & $\ldots$ & 2.1 \\
\hline$H S T^{\circ}$ & 1996212 & $55 . \pm 4$ & $72 . \pm 5.2$ & $\ldots$ & $\ldots$ & $(370 .-1400)$. & $\ldots$ & 1.3 \\
\hline$H S T$ & 1999278 & 72.p & 111. & $\leqslant 30 .{ }^{q}$ & $\ldots$ & $\ldots$ & $\ldots$ & 1.5 \\
\hline$H S T$ & 2012313 & 64. & $76 .^{r}$ & $\leqslant 20$. & $\cdots$ & $\ldots$ & $\ldots$ & 1.2 \\
\hline$H S T$ & 2012365 & 41. & $77 . \mathrm{s}$ & 45. \pm 31 & $\ldots$ & $\cdots$ & $\cdots$ & 1.9 \\
\hline
\end{tabular}

Notes.

a Observatory.

b $\mathrm{O} I\left({ }^{3} P-3 s^{3} S_{o}\right)$ at $1304 \AA$ brightness in Rayleighs.

c $\mathrm{O}$ I $\left({ }^{3} P-3 s^{5} S_{o}\right)$ at $1356 \AA$.

${ }^{\mathrm{d}} \mathrm{H}$ I resonance line $1216 \AA$.

${ }^{\mathrm{e}}$ Line-of-sight abundance $\left(10^{12}\right.$ atoms or molecules $\left.\mathrm{cm}^{-2}\right)$.

${ }^{\mathrm{f}}$ See the text.

g Present work, trailing surface.

${ }^{\mathrm{h}}$ Read 2001012 as 2001 DOY 012.

${ }^{\mathrm{i}}$ Average brightness over disk inside $1.15 R_{E}$.

${ }^{\mathrm{j}}$ Inferred from $\mathcal{I}\left({ }^{5} S\right)$ and Kliore et al. (1997) electron environment. Opacity abundance from analysis of $\mathcal{I}\left({ }^{3} S\right)$ is also $4.7 \times 10^{12} \mathrm{~cm}^{-2}$.

${ }^{\mathrm{k}}$ Hall et al. (1995), trailing surface; based on Table 1 of Hall et al. (1998).

${ }^{1}$ Average brightness over disk.

${ }^{m}$ From Hall et al. (1998).

${ }^{\mathrm{n}}$ Hall et al. (1998), leading surface.

${ }^{\circ}$ Hall et al. (1998), trailing surface.

p O I calculated from Figure S5 (A) of Roth et al. (2014), average brightness over $1.15 R_{E}$ disk, see the text.

q Averaged H Ly $\alpha$ emissions as reported by Roth et al. (2014).

${ }^{\mathrm{r}}$ O I calculated from Figure S5 (B) of Roth et al. (2014).

s O I calculated from Figure S5 (C) of Roth et al. (2014).

O I multiplet brightnesses are disk averaged values. The listed emission brightnesses in Table 3 for the observations reported by Roth et al. (2014, see Table S1) are similar but not equal to the values derived from the flux information in Roth et al. (2014), given in Table 3. As stated in Table 3, the flux from the Roth et al. (2014) measurements are extracted from Roth et al. (2014, Figure S5). The O I disk averaged emission values do not show strong variation from 1994 to the present according to this analysis. The brightness quantities in Table 3 are derived from flux values. The disk-averaged emissions in the plume episode show no evidence of a global impact on the atmosphere (Table 3), comparing the 2012 DOY 365 results with 2012 DOY 313. Observation to observation variability in the HST results is mainly in the $\mathcal{I}\left({ }^{3} S\right)$ emission, probably caused by significant variable solar reflection contamination, which is not present for the $\mathcal{I}\left({ }^{5} S\right)$ feature.

\subsection{Spectral Model Calculations}

The model calculations in the reduction of the spectra shown in Figures 3 and 6 are based on the methodology described in Shemansky \& Smith (1981) using updated physical parameters where necessary. The model code calculates statistical equilibrium in the gas with the applied fixed forcing of the system. The collision strengths are established on the basis of the principle of detailed balance so that at very high density, the state populations approach a Maxwell-Boltzmann distribution. The calculations are non-LTE statistical equilibrium. The $\mathrm{O} I$ architecture used in fitting the spectrum of Figure 6 contains 54 states; calculations take place at the electron orbital level, predicting emission in 887 transitions. Other species in the model calculations are developed to a similar level (Shemansky \& Smith 1981). Emission in the O I system depends on the combined effects of solar flux and electron impact. In general, there is dependence on electron density. The rate data given in Figure 10 for $\mathrm{O}$ I are for ambient electron density $[\mathrm{ec}]=10^{4} \mathrm{~cm}^{-3}$. The solar flux model, which combines solar spectral observations with solar plasma modeling, is required to be at high resolution $(\sim 200,000-600,000)$ in order to avoid errors in the fluorescence calculations (Killen et al. 2009).

\subsubsection{Limits on Neutral Species Based on Spectral Analysis}

The limits on $\mathrm{H}_{\mathrm{I}}$ and $\mathrm{H}_{2}$ in the Europa plasma sheet and atmosphere are less definitive than those determined from the plasma sheet properties, but these quantities contribute to the accumulated evidence. O I or other neutrals have not been detected in emission from the plasma sheet. The impact of these results has relevance to previous model calculations of atmospheric structure and consequent rates of mass loading at $9.4 R_{J}$. The atmospheric model calculations by Saur et al. (1998) is compared to the present results in Table 4. The Saur et al. (1998) model is based on an assumed atmosphere dominated by $\mathrm{O}_{2}$. Smyth \& Marconi (2006) provide a model based on proton surface sputtering, which removes molecules into the vacuum by the inefficient process of nuclear momentum transfer, whereas multiply charged ions sputter by the exchange and dissociative recombination process (Shemansky 2003). The physical chemistry applied by Smyth \& Marconi (2006) produces an atmosphere dominated by $\mathrm{O}_{2}$, with $\mathrm{H}_{2}$ the second most abundant species. Species limits from the present work are compared to previous model results in Table 5. The limit on $\mathrm{H}_{2}$ abundance established here is $\sim 100$ times below the Smyth \& Marconi (2006) model calculations in the plasma sheet at Europa (Table 5). For future reference, Figure 8 shows the upper limits on $\mathrm{H}_{2}$ brightness in the EUV/FUV calculated for 

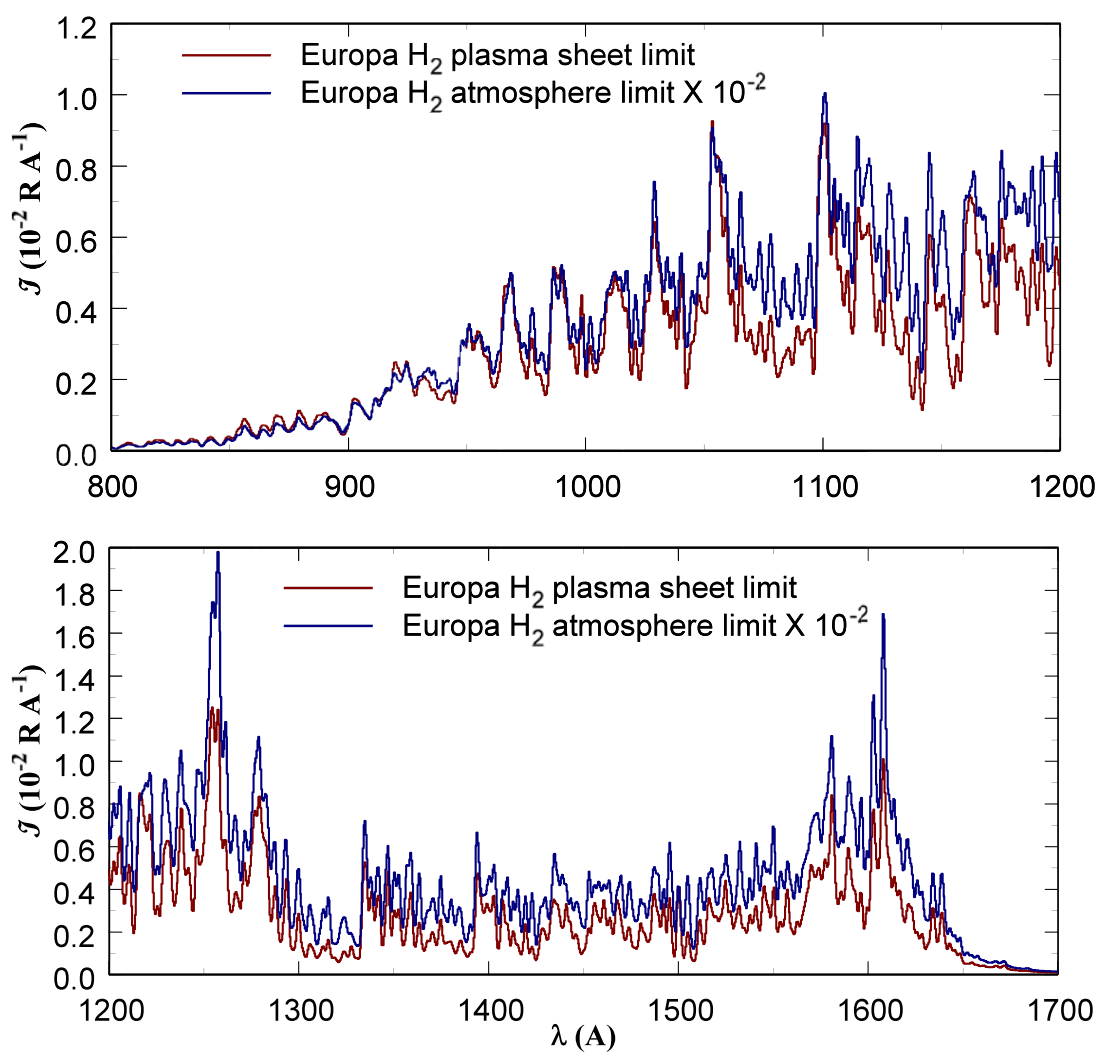

Figure 8. Model spectra of electron and solar forced $\mathrm{H}_{2}$ emission in the EUV/FUV region, using parameters for the Europa atmosphere and plasma sheet. The calculations are made for a nominal $2 \AA$ FWHM Gaussian point-spread function, giving absolute scale upper limits in differential brightness $\left(R \AA^{-1}\right)$. The model includes rotational levels limited only by the dissociation limit in most electronic state potentials used in the calculation (see Liu et al. 2007). Details of the solar spectrum used in the calculation are provided by Killen et al. (2009).

(A color version of this figure is available in the online journal.)

Table 4

Critical Plasma Sheet and Gas-surface Reaction Rates

\begin{tabular}{lccccccccc}
\hline \hline$\rho \mathrm{O}_{23}{ }^{\mathrm{a}}$ & $\rho \mathrm{O}_{32}{ }^{\mathrm{b}}$ & {$[N \ell]^{\mathrm{c}}$} & $N \ell T^{\mathrm{d}}$ & $N \ell T^{\mathrm{e}}$ & $S(N \ell)^{\mathrm{f}}$ & $S(N \ell)^{\mathrm{g}}$ & $\bar{Y}_{S}{ }^{\mathrm{h}}$ & $\mathcal{F ~ O}_{3}{ }^{\mathrm{i}}$ & ${\overline{S_{Y}}}^{\mathrm{j}}$ \\
\hline 94. & 3.2 & 3. & 4.6 & 90. & 4.5 & 200. & 3.0 & 0.45 & 1.3 \\
\hline
\end{tabular}

Notes.

a Probability $\left(\rho\left(\times 10^{-9} \mathrm{~s}^{-1}\right)\right)$ for electron impact ionization of O III, $\operatorname{rr}(11)$.

b Probability for dielectronic recombination of O IV, $\operatorname{rr}(12)$.

c Limiting neutral density $\left(\mathrm{cm}^{-3}\right)$ to allow steady state of plasma sheet O IV population.

${ }^{\mathrm{d}}$ Limit to total number of neutral atoms in the plasma sheet $\left(\times 10^{32}\right)$.

e Mauk et al. (2003).

${ }^{\mathrm{f}}$ Limiting source rate of neutral particles maintaining the plasma sheet $\left(\times 10^{25} \mathrm{~s}^{-1}\right)$.

g Saur et al. (1998).

h Sputtering yield of neutral atoms by plasma sheet O IV ions.

${ }^{\mathrm{i}}$ Flux of O IV ions into the Europa surface $\left(\times 10^{8} \mathrm{~cm}^{-2} \mathrm{~s}^{-1}\right)$.

${ }^{\mathrm{j}}$ Flux of surface sputtered atoms into the vacuum $\left(\times 10^{8} \mathrm{~cm}^{-2} \mathrm{~s}^{-1}\right)$.

the volumetric conditions in the plasma sheet and the Europa atmosphere. The $\mathrm{H}_{2}$ model calculations include rotational levels extending to $J=25$ (Liu et al. 2007). The calculations are carried out for the combined forcing of solar flux and electron impact, in statistical (non-LTE) equilibrium. Differences in the spectra are caused by electron density and temperature, and the interaction of solar flux with significantly different ground state vibration-rotation populations. Model details will not be provided. Outward diffusion of atomic oxygen from the Io torus does not reach the orbit of Europa in measurable quantities (Smyth \& Shemansky 2005). The excitation of neutrals by the inwardly diffusing hot magnetosphere population (Paonessa
Table 5

Neutral Species Abundance $\left(\times 10^{12} \mathrm{~cm}^{-2}\right)$

\begin{tabular}{|c|c|c|c|c|c|c|c|}
\hline \multirow[t]{2}{*}{ Species } & \multicolumn{2}{|c|}{ Present } & \multicolumn{2}{|c|}{ Smyth $^{\mathrm{a}}$} & \multirow{2}{*}{$\begin{array}{l}\text { Saur }^{b} \\
\text { Europa }\end{array}$} & \multirow{2}{*}{$\begin{array}{c}\text { Ip }^{\mathrm{c}} \\
\text { Europa }\end{array}$} & \multirow{2}{*}{$\begin{array}{l}\text { Roth }^{\mathrm{d}} \\
\text { Europa }\end{array}$} \\
\hline & Plasma & Europa & Plasma & Europa & & & \\
\hline$[\mathrm{O} \mathrm{I}] \ell$ & $\leqslant 0.23$ & 4.7 & 5. & 1.2 & $\cdots$ & $\cdots$ & 1. \\
\hline$[\mathrm{HI}] \ell$ & $\leqslant 0.18$ & & $\cdots$ & 0.076 & $\ldots$ & $\ldots$ & $\ldots$ \\
\hline$\left[\mathrm{H}_{2}\right] \ell$ & $\leqslant 1$. & $\leqslant 4.5$ & $100 .^{\mathrm{e}}$ & 77. & $\ldots$ & $\ldots$ & $\ldots$ \\
\hline$\left[\mathrm{O}_{2}\right] \ell$ & $\ldots$ & $\cdots$ & $\cdots$ & 450. & 500. & $200 .-2000 .^{f}$ & 350. \\
\hline$\left[\mathrm{H}_{2} \mathrm{O}\right] \ell$ & $\ldots$ & $\cdots$ & $\cdots$ & 2.3 & $\cdots$ & $\ldots$ & 17. \\
\hline
\end{tabular}

Notes.

a Smyth \& Marconi (2006).

b Saur et al. (1998).

c Ip et al. (1998).

d Roth et al. (2014).

${ }^{\mathrm{e}}$ Line of sight through Europa in orbital plane.

${ }^{\mathrm{f}}$ Heterogeneous magnetosphere surface sputtering, source rate $3 \times 10^{26} \mathrm{O}_{2} \mathrm{~s}^{-1}$.

1982) is negligible, although ambient electron heating by this flux is significant. Another investigation (Lagg et al. 2003) has reported finding evidence of significant neutral populations near Europa as interpreted from observed perturbations of pitch angle distributions of magnetosphere ions, in conflict with the present results. The production of a surface sputtered atmosphere at Europa has been discussed by Ip et al. (1998), Cooper et al. (2001), Cassidy et al. (2013), and Sittler et al. (2013).

Table 3 shows $\mathrm{O}_{2}$ abundances in Column 7 required if the $\mathrm{O}$ I emission is produced by dissociative excitation (Hall et al. 1995). These values are 100-1000 times larger than the present derived abundance for an O I atmosphere. Given an O I 
Table 6

Nominal Europa Atmosphere

\begin{tabular}{lcccccc}
\hline \hline$[\mathrm{OI}]^{\mathrm{a}}$ & {$[\mathrm{O} \mathrm{II}]$} & {$[e]$} & $T_{g}(\mathrm{~K})$ & $H(\mathrm{OI})^{\mathrm{b}}$ & $H(e, \mathrm{O} \mathrm{II})$ & {$[\mathrm{OI}] /[e]$} \\
\hline $1.5^{\mathrm{c}}$ & 0.1 & 0.1 & 800. & 300. & 240. & 15. \\
$13 .^{\mathrm{d}}$ & 0.23 & 0.23 & 150. & 31. & 31. & 56. \\
\hline
\end{tabular}

Notes.

a Densities ([]) $\times 10^{5} \mathrm{~cm}^{-3}$ at the solid surface.

b Scale height $(H)$ in $\mathrm{km}$.

${ }^{c}$ Present observation based results.

d Present preliminary model.

atmosphere the major ionosphere ion must be $\mathrm{O}$ II. The presence of $\mathrm{O}$ II in the Europa atmosphere has not been directly observed in the UVIS exposures. It is clear that the plasma sheet densities, measured in 2001 DOY 012, peak broadly around the location of Europa, but a peak in $\mathrm{O}$ II emission confined to the pixel containing the Europa image has not been detected above the background emission from the plasma sheet. The upper limit in Column 8 of Table 3 is close to the abundance expected if the major ion is O II. The electron vertical profile has been measured in Galileo radio occultations by Kliore et al. (1997). The mean of six occultations provided a vertical profile showing electron density peaking at the surface with a value $[\mathrm{ec}] \sim 9000 \mathrm{~cm}^{-3}$. The electron abundance (Kliore et al. 1997) is close to the limit given for $\mathrm{O}$ II in Table 3. The neutral/ion mixing ratio at the surface from this analysis is then $[N] /[e] \sim 15$, leading to the conclusion that the Europa atmosphere is actually a weakly ionized plasma with a density maximum at the surface. There are no atomic kinetic collisions in this nominal atmosphere; the primary collision partners are electrons. The atmospheric plasma on Europa can be compared to the state of the Enceladus plasma torus in the Saturn system. Model calculations of an energy equilibrated $\mathrm{H}_{2} \mathrm{O}$ sourced system at Enceladus produces $[N] /[e] \sim 60$, with $T_{e} \sim 16,000 \mathrm{~K}$ (Yoshii 2011). The state of the gas in these systems is limited by the energy available in the magnetospheric structure, and the mass insertion rate. In this case the Europa atmosphere is a higher temperature, more heavily ionized plasma than the Enceladus torus.

\section{ATMOSPHERIC MODELS}

The most recent published atmospheric models for Europa are based on the interpretation that the disk averaged emissions $\mathcal{I}\left({ }^{3} S\right)$ and $\mathcal{I}\left({ }^{5} S\right)$ are from dissociatively excited $\mathrm{O}_{2}$ (Saur et al. 1998; Smyth \& Marconi 2006). A model based on an O I dominated atmosphere, developed from the Liang et al. (2005) model for Callisto is presented here. The source of $\mathrm{CO}_{2}$ in the model is suppressed and overall source rates reduced, leaving an atmosphere dominated by $\mathrm{O}_{\mathrm{I}}$ and $\mathrm{HI}$, derived from $\mathrm{H}_{2} \mathrm{O}$ photolysis. The source process is not necessarily an accurate description of how the atmosphere has developed, but it provides a more realistic structure for comparison to observation. Figure 9 shows the vertical distribution of the primary species. The dominant ion is $\mathrm{O}$ II, with a vertical abundance within a factor of two of the Kliore et al. (1997) measurements. The densities and scale heights are shown in Table 6, in comparison to the observational results discussed here. The abundance of $\mathrm{H} \mathrm{I}$ in the model would not be detectable in the observations to date. The abundance of $\mathrm{O}_{2}$ (Figure 9) in the model, however, is roughly a factor of two to three too large for compatibility with the present observations. The major loss of $\mathrm{O}_{\mathrm{II}}$ in the model is through reactions $\operatorname{rr}(9)$ and $\operatorname{rr}(10)$. An $\mathrm{O}_{\mathrm{II}}$ ionosphere is unsustainable on Callisto because of the fast $\operatorname{rr}(10)$. This is not an issue for Europa. The scale heights in the model are much smaller than the observation derived quantities, because the source function is cold. The model assumes that $\mathrm{H}_{2} \mathrm{O}$ is extracted from the surface by nuclear sputtering. This may not be an accurate description if exchange processes play a significant role, in which the particles diffused into the vacuum are mainly atomic (Shemansky 2003).

\subsection{Rate Processes}

The conclusion in earlier work that the Europa atmosphere was primarily $\mathrm{O}_{2}$ was based on an observed emission ratio $\mathcal{I}\left({ }^{5} S\right) / \mathcal{I}\left({ }^{3} S\right)$ consistently greater than one. The observed values for $\mathcal{I}\left({ }^{5} S\right) / \mathcal{I}\left({ }^{3} S\right)$ range from 1 . to 2.2 (Table 3$)$. The reactions producing emission in the two multiplets that require consideration here are as follows:

$$
\begin{gathered}
e+\mathrm{O}_{2}(X) \rightarrow e+\mathrm{O}^{*}+\mathrm{O}^{*} \\
e+\mathrm{O}_{2}(X) \rightarrow e+\mathrm{O}\left({ }^{3} P\right)+\mathrm{O}\left(3 s^{5} S_{\mathrm{o}}\right) \\
e+\mathrm{O}_{2}(X) \rightarrow e+\mathrm{O}\left({ }^{3} P\right)+\mathrm{O}\left(3 s^{3} S_{\mathrm{o}}\right) \\
e+\mathrm{H}_{2} \mathrm{O}(X) \rightarrow e+\mathrm{H}(2 s, 2 p)+\text { products } \\
e+\mathrm{H}_{2} \mathrm{O}(X) \rightarrow e+\mathrm{O}\left(3 s^{5} S_{\mathrm{o}}\right)+\text { products } \\
e+\mathrm{H}_{2} \mathrm{O}(X) \rightarrow e+\mathrm{O}\left(3 s^{3} S_{\mathrm{o}}\right)+\text { products } \\
e+h v+\mathrm{O}\left({ }^{3} P\right) \rightarrow e+\mathrm{O}\left(3 s^{5} S_{\mathrm{o}}\right) \\
\left.e+h v+\mathrm{O}^{3} P\right) \rightarrow e+\mathrm{O}\left(3 s^{3} S_{\mathrm{o}}\right) \\
\mathrm{O}^{+}+\mathrm{O}_{2} \rightarrow \mathrm{O}^{2} \mathrm{O}_{2}^{+} \\
\mathrm{O}^{+}+\mathrm{CO}_{2} \rightarrow \mathrm{CO}^{+} \mathrm{O}_{2}^{+} \\
e+\mathrm{O}^{2+} \rightarrow \mathrm{O}^{3+}+2 e \\
e+\mathrm{O}^{3+} \rightarrow \mathrm{O}^{2+} \\
\mathrm{O}^{3+} \rightarrow \text { diffusive loss } \\
\mathrm{O}^{3+}+\mathrm{N}^{*} \rightarrow \mathrm{O}^{2+}+\mathrm{N}^{+*}
\end{gathered}
$$

where $\mathrm{N}^{*}$ refers to a neutral atom, atomic ion, or neutral molecule. Rate coefficients critical to the present discussion are given in Figure 10. As expected, reaction (rr) rr(1) (derived from Itikawa 2003) has the most aggressive rates, and it is this reaction that forces high mass loading of the plasma sheet if the Europa O I emissions are assumed to be caused by $\operatorname{rr}(2)$ and $\operatorname{rr}(3)$. The assumption of an $\mathrm{O}_{2}$ dominated atmosphere introduces three difficulties. (1) The ratio $\mathcal{I}\left({ }^{5} S\right) / \mathcal{I}\left({ }^{3} S\right)$ from $\operatorname{rr}(2)$ and $\operatorname{rr}(3)$ ranges from 3.5 at $T_{e}=50,000 \mathrm{~K}$ to 2.6 at $T_{e}=250,000 \mathrm{~K}$, out of range of the observations (Table 3 ) according to the current rates shown in Figure 10. (2) Based on the Saur et al. (1998) calculations using the $H S T$ observed emissions, an injection rate of $2 \times 10^{27} \mathrm{O}$ I atoms s${ }^{-1}$ is indicated for the plasma sheet. This is not compatible with the state of the observed plasma. (3) The analysis of the UVIS observations described above, taking opacity into account, fits the observed spectrum and $\mathcal{I}\left({ }^{5} S\right) / \mathcal{I}\left({ }^{3} S\right)$ ratio with the present model for $\operatorname{rr}(7)$ and $\operatorname{rr}(8)$ (Figure 6). Roth et al. (2014), indicating emissions that are consistent with $\operatorname{rr}(4)$ and $\operatorname{rr}(6)$, is the only published work showing evidence of $\mathrm{H}_{2} \mathrm{O}$ in the near Europa environment (see Figure 10). The rates for dissociative excitation of $\mathrm{H}_{2} \mathrm{O}$ shown in Figure 10 are derived 


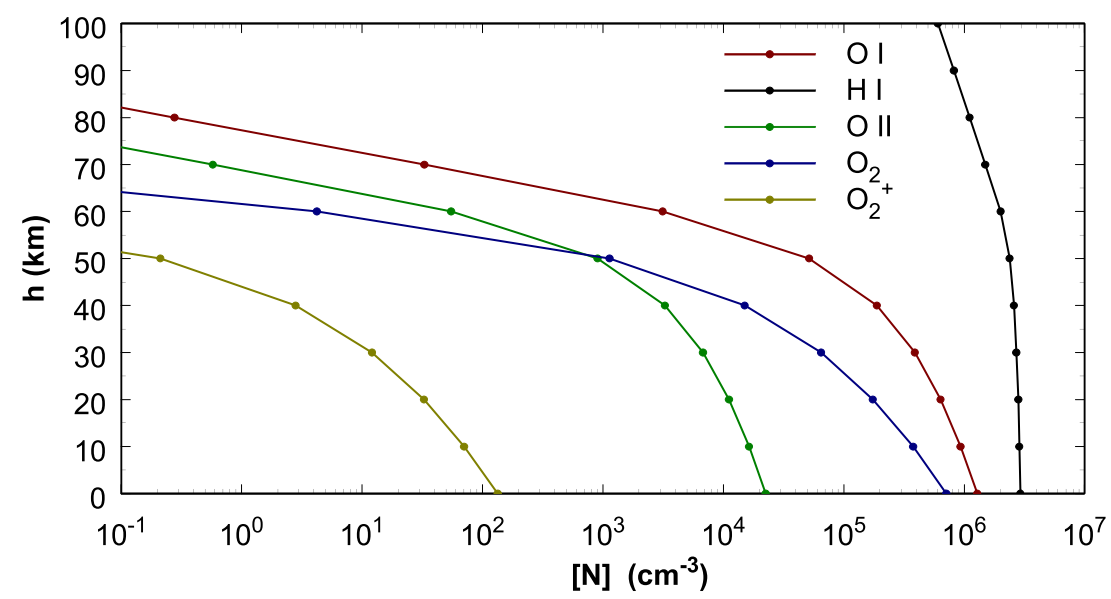

Figure 9. Europa model atmosphere based on the Liang et al. (2005) model for Callisto, in which the $\mathrm{CO}_{2}$ source is suppressed to reflect conditions at the surface of Europa. The oxygen in this model is derived from $\mathrm{H}_{2} \mathrm{O}$ extracted from the surface cold, with recycling, leaving a cold $\mathrm{O}$ I atmosphere. The legend identifies the species in the plot, $\mathrm{O}_{\mathrm{I}}, \mathrm{H}_{\mathrm{I}}, \mathrm{O}_{\mathrm{II}}, \mathrm{O}_{2}$, and $\mathrm{O}_{2}^{+}$.

(A color version of this figure is available in the online journal.)

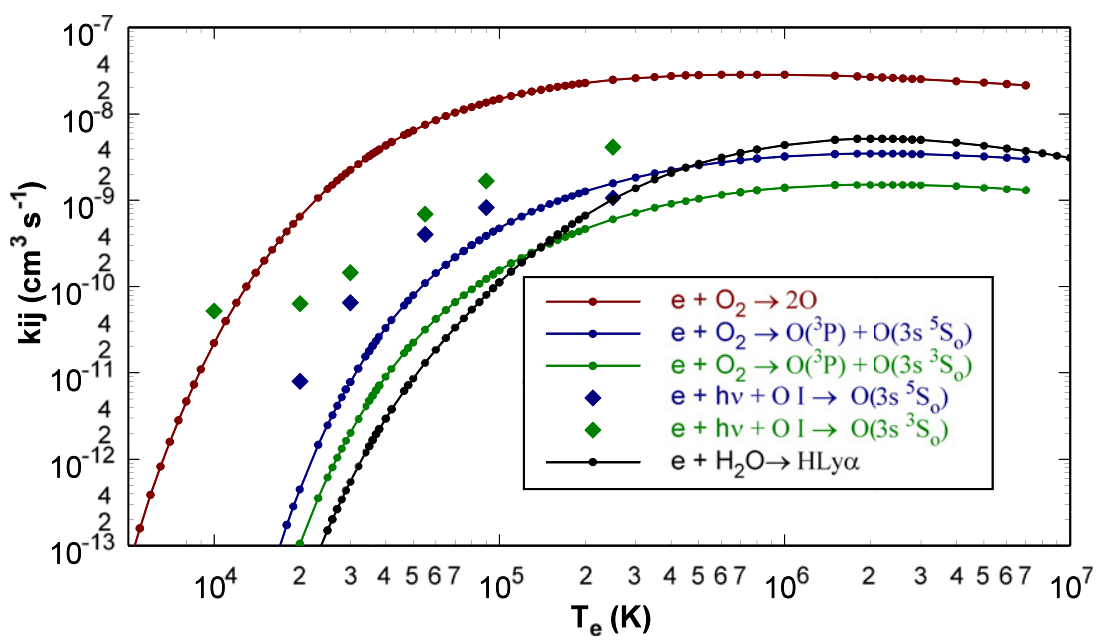

Figure 10. Rate coefficients for reactions $\operatorname{rr}(1)$ through $\operatorname{rr}(4), \operatorname{rr}(7)$, and $\operatorname{rr}(8)$ (see the text). The forcing in $\operatorname{rr}(7)$ and $\operatorname{rr}(8)$ includes a high-resolution solar flux model (Killen et al. 2009). The latter two reactions have electron density dependence; these calculations are for [ec] $=10^{4} \mathrm{~cm}^{-3}$, where [ec] refers to the ambient electron population. A hot heterogeneous electron population, $[\mathrm{eh}]=2 \mathrm{~cm}^{-3}\left(T_{\mathrm{eh}}=10^{7} \mathrm{~K}\right)$ is included in $\operatorname{rr}(7)$ and $\operatorname{rr}(8)$, and rates are for optically thin gas. The solar flux and $[\mathrm{eh}]$ acquire greater influence at low ambient electron temperatures.

(A color version of this figure is available in the online journal.)

from Morgan \& Mental (1974). The observations examined here show no evidence of $\mathrm{H}$ Ly $\alpha$ emission in the plasma sheet or Europa atmosphere. The stochastic process constituting the observations listed in Table 3, does not show evidence of deviation from an overall stationary state in the Europa atmosphere. The imaging of the emission on the Europa disk does show variability, described as auroral activity (Roth et al. 2014), and general evidence of a variable Europa environment has been discussed (Intriligator \& Miller 1982; Russell 2005) but evidence for strong localized events that significantly affect the Europa global atmosphere, is absent in the present assessment. The Roth et al. (2014) analysis of the plume observation of 2012 DOY 365 did not develop an estimate of mass loading of the magnetosphere. The plume altitude limited at $200 \mathrm{~km}$, suggesting that a large fraction of the gas did not reach escape velocity and did not significantly affect the plasma sheet.

\subsection{Nominal Atmosphere}

Table 6 shows the nominal Europa model atmosphere emerging from the present work. Examination of the details of en- ergy balance and rate processes are beyond the scope of this preliminary work. Some general conclusions, however, can be drawn. The ionosphere, which Kliore et al. (1997) have shown interfaces with the solid surface, will be sustained by both electron impact and charge capture by plasma sheet ions, which penetrate to the surface. Substantial recycling between solid state and gas is expected, with sputtering and dissociative excitation to neutral atoms through multicharged particle penetration of the surface (Aumayr \& Winter 2004; Shemansky 2003). It is evident, however, that Europa does not easily deliver mass to the magnetosphere. The strongest rate processes are likely to be solid to gas recycling.

\subsection{The Physics of Gas-Surface Interaction}

Calculations to date of processes extracting gas from reactions at the surface of Europa have been limited mainly to consideration of singly charged magnetospheric ions sputtering the surface mainly through nuclear momentum transfer (see the discussion by Ip 1996; Ip et al. 1997, 1998, in which molecules are delivered into the vacuum by ion impact). Given the Hall et al. 
(1995) results, it has been assumed that the primary process is sputtering of $\mathrm{O}_{2}$ from ion impact (Saur et al. 1998), supported by the observed phenomenon in laboratory work with solid $\mathrm{H}_{2} \mathrm{O}$ (Johnson et al. 1983; see Teolis et al. 2005). Gas-surface interaction, however, has complexities that need consideration beyond nuclear momentum transfer. In this case, the top layers of surface of Europa is significantly deviated from pure solid $\mathrm{H}_{2} \mathrm{O}$ through long-term weathering and is certainly not understood. In addition to this consideration, the Europa ionosphere is in contact with the surface, and presumably plays a significant role in the range of rate processes affecting the state of the surface. The range of ion-surface impact interactions has also not been fully considered, and the assumption that this process dominantly removes molecules into the vacuum is not correct. Substantial research has been carried out in this discipline, particularly in the era of nuclear fusion research in the 1970s. The basic physics is now generally understood, although specific rates are not extensively determined.

Energy loss by impacting ions is described (Townsend et al. 1976) as three approximately separable components; nuclear, electronic, and exchange, as shown here in $\operatorname{rr}(15)$ :

$$
\left(\frac{d E}{d x}\right)_{\mathrm{loss}}=\left(\frac{d E}{d x}\right)_{\mathrm{nuc}}+\left(\frac{d E}{d x}\right)_{\mathrm{elect}}+\left(\frac{d E}{d x}\right)_{\mathrm{exch}} .
$$

The first two terms are generally dominant if the impactor is singly charged. This is the case in Europa atmospheric modeling. If the impacting ion is multiply charged, a significant amount of energy per ion is invested, and this energy is released in extremely fast reactions at the surface in cascading electron capture that results exclusively in molecular dissociation and the emission of soft X-rays. The process takes place very close to the top surface layer, efficiently delivering the atomic products into the vacuum. Fast charge exchange reactions producing $\mathrm{X}$-ray emission were discovered in comet comae by Lisse et al. (2001) forced by highly charged solar wind ions, an important aspect of comet science.

The Galileo energetic particle detector (EPD) experiment obtained measurements showing that the heterogeneous magnetospheric flux at Europa is dominated by sulfur and oxygen ions (Ip et al. 1997; Section 3.1.1). It is clear that these components mainly originate at the Io plasma torus. The observations reported here show that most of the plasma sheet ions at Europa originate at the Io plasma torus. Given the low densities and high electron temperatures in the magnetosphere, the ions in the diffusion process will increase in charge level as a function of time, before they are delivered to objects such as Europa. The charge levels in the ambient plasma in the equatorial region certainly increase during the diffusion time to the Europa plasma sheet (Tables 1 and 2). The ions involved in the sputtering process will certainly be $\mathrm{s}^{n+}$ and $\mathrm{O}^{n+}$ with $n>2$. On this basis, the third term in $\operatorname{rr}(15)$ is expected to be dominant. Ions in the plasma sheet will also contribute. Model calculations exploring the rate processes related to the third term of $\operatorname{rr}(15)$ are needed to fully explore atmospheric development.

\section{SENSITIVITY OF THE PLASMA SHEET AT $9.4 R_{J}$ TO NEUTRAL GAS}

The state of the plasma sheet established here depends on a very low mass loading rate to retain significant ion charge levels. A qualitative assessment from the nature of the source of the ion population indicates that in the transport time for delivery of sulfur ions from $5.9 R_{J}$ to $9.4 R_{J}$, the plasma electron volumetric energy increases from $75,000 \mathrm{~K}$ to $250,000 \mathrm{~K}$ against losses to collisional radiative reactions, ionization, and dielectronic recombination (Shemansky 1988). It is evident that the heterogeneous magnetospheric electrons deposit a net large amount of energy into the ambient population (electron-electron Coulomb interaction) to raise the energy density of the resident electrons against exponentially rising loss rates (Shemansky 1988). This is a qualitative indicator that mass loading from Europa must on average be at a very low level. Without carrying out a detailed physical chemistry energy balanced calculation such as Shemansky (1988) and Yoshii (2011), approximate limiting rates and timescales can be established to provide values of core parameters. Table 4 lists the pivotal quantities. The measurement of O IV at $9.4 R_{J}$ provides one of the longer time constants. The conversion of $\mathrm{O}$ III to $\mathrm{O}$ IV by electron impact $(\operatorname{rr}(11))$ has a characteristic time of 124 days (Table 4). This process takes place in competition with diffusive loss $(\operatorname{rr}(13))$, dielectronic recombination $(\operatorname{rr}(12))$, and charge capture from neutral atoms $(\operatorname{rr}(14))$ and lower order ions. The probability for $\operatorname{rr}(11) \rho 11=9.3 \times 10^{-8} \mathrm{~s}^{-1}$ must be more rapid than charge capture (Aumayr \& Winter 2004) or other losses in order to build population as it does during transport from 5.9 $R_{J}$. This requires a mean neutral density in the plasma sheet of less than $3 \mathrm{~cm}^{-3}$. Neutral emission upper limits are at a level of $\sim 3 \mathrm{~cm}^{-3}$. The rate of neutral injection at this level in the plasma sheet is $\sim 4 \times 10^{25}$ atoms s ${ }^{-1}$ (Table 4 ). This is a crude estimate of a sustainable level of mass loading for the plasma sheet. Given that the electron density and temperature measurements in the Europa plasma sheet at the time of Voyager, Galileo, and Cassini constitute a stochastic process in addition to the Europa atmospheric emission results, the indication is that the observations on a timescale of years constitute an approximate steady state on a long timescale. The injection of atoms at a rate 1000 times the above mean value may take place for a limited interval on a frequency scale of years, but the mean value must not exceed the $\sim 4 \times 10^{25}$ atoms s ${ }^{-1}$ rate. The evidence for large events in the Europa atmosphere does not appear in the stream of stochastic samples to date.

The influence of plasma sheet ions on the generation of atmospheric gas is a factor to consider, given that multiply charged ions are involved. The sputtering of solid surfaces is determined by momentum transfer, electronic excitation, and exchange reactions. The last process has generally received little attention because the process has a small role in singly ionized ion impacts. Multiply charged ions, however, can contain a significant amount of internal energy that is delivered to the solid molecules in charge capture and subsequent emission in multiple X-ray photon deposition into the solid (Aumayr \& Winter 2004; Shemansky 2003). Charge capture processes in the solar system have been discussed in Shemansky (2003). The plasma sheet ion impact energies at the surface of Europa are only at the low keV level, and only exchange reactions can contribute significantly to sputtering. The exchange sputtering process is one of ionization by the multiply charged ion with simultaneous photon emission. The photo electrons released produce atomic products in dissociative recombination, with enough kinetic energy to diffuse into the vacuum. O IV has enough energy to contribute to the sputtering process. Magnetospheric protons, at much higher kinetic energy sputter through momentum transfer. Efficiencies are discussed in Shemansky (2003). The calculated rates for the $\mathrm{O}$ IV sputtered contribution given in Table 4 do not include kinetic energy distributions, but the values indicate that 
plasma sheet ions may have a significant contribution to the Europa atmosphere. Sputtering rates have been discussed by Ip (1996). The heterogeneous flux of magnetospheric ions, based on the Galileo EPD experiment, contains ions in the rough ratio $\mathrm{H}^{+}: \mathrm{S}^{n+}: \mathrm{O}^{n+} \sim 6: 4: 0.4$ (Ip et al. 1998) with relative sputtering efficiency for $n=1 \sim 0.1: 3: 1$ (Ip et al. 1998). The Galileo EPD identified the ion species in the magnetosphere, but could not determine charge. The ion charge is a critical factor in the determination of exchange sputtering rates. The result of primary importance in the Galileo EPD measurements is the fact that the dominant ion was $\mathrm{s}^{n+}$. The value of $n$ is an important parameter (Shemansky 2003) for the determination of sputtering rates at Europa. The determination of $n$ depends on the mean lifetime of the diffusion process to the outer magnetosphere and adiabatic inward drift. Given a diffusion time of $\sim 100$ days between 5.9 and $9.4 R_{J}$ for ambient ions, a lifetime for the heterogeneous ions of at least $\sim 1000$ days is not unreasonable, so that $n \geqslant 4$ could be expected for the sulfur ions. The charged heterogeneous ions may therefore be effective in contributing to the maintenance of the Europa atomic atmosphere through the exchange process at the surface.

The current calculated population of neutrals in the plasma sheet can be compared to the Mauk et al. (2003) result from energetic neutral particle imaging, and the Saur et al. (1998) model source rates, given in Table 4. The rates by Saur et al. (1998) are much larger because of the assumption (Figure 10) that the atmosphere was dominated by $\mathrm{O}_{2}$. Analysis of Galileo EPD measurements of equatorial proton pitch angle distributions (Lagg et al. 2003) indicate lower energy protons (80-220 keV) are depleted at $90^{\circ}$ near Europa. Lagg et al. (2003) interpreted this as indicating the presence of 20-50 neutrals $\mathrm{cm}^{-3}$. This is a factor of $\sim 3$ below the Mauk et al. (2003; Table 4) density, but still an order of magnitude above the limit established here at Cassini encounter.

\section{CONCLUSIONS AND DISCUSSION}

1. Deep exposures using the Cassini UVIS EUV spectrograph have revealed a low-density plasma at the Europa orbit, composed mainly of ions originating in the Io plasma torus. This is a predictable finding because the radial electron density distribution measured previously using the Voyager and Galileo missions produced similar densities and temperatures to the values in the present analysis. No neutral atomic emissions were detected, and upper limits from approximate plasma analysis and the emission results indicate a mean neutral density of $3 \mathrm{~cm}^{-3}$ in the plasma sheet. There is an indication that Europa contributes oxygen to the plasma. Mass loading of the plasma sheet is estimated to be $\sim 5 \times 10^{25}$ atoms s ${ }^{-1}$, a factor of 40 below the Saur et al. (1998) $\mathrm{O}_{2}$ model (Table 4).

2. The atomic oxygen multiplet emissions from Europa identified in previous observations beginning in 1994 have been misinterpreted as dissociative excitation of an atmosphere dominated by $\mathrm{O}_{2}$. The consequence of this earlier conclusion was the adoption of a model that inserted significant mass loading into the plasma sheet at the Europa orbit. The present analysis of the Cassini UVIS FUV measurements indicates that the emission spectrum averaged over the Europa disk is electron-excited atomic oxygen. The atmosphere at Europa in the present analysis is atomic oxygen at a density two orders of magnitude below the previous $\mathrm{O}_{2}$ dominated model.
3. The heterogeneous hot component of the magnetospheric plasma deposits most of the energy required to maintain the Io plasma torus. The energy density at the orbit of Europa is at a similar level and, given mass loading, could support a plasma torus on the same scale. This has never been observed. The energy passing through the plasma at the Io torus (Table 1) is two orders of magnitude greater than in the plasma sheet at Europa at the time of Voyager encounter in 1979 and at Cassini encounter in 2001. Given the stochastic remote sensing evidence, mass loading at the Europa orbit is not consistent with a geophysically active body.

The overall impact of the present work on the assessment of the level of geophysical activity is to indicate that the evidence for vents releasing gas into the atmosphere is at best tenuous. Russell (2005) has pointed out that periodic plasma enhancements from Pioneer 11 measurements were interpreted by Intriligator \& Miller (1982) as a continuous plume at Europa. The phenomenon was, however, found to be non-repeatable in work with Galileo (Russell 2005). These (Intriligator \& Miller 1982) results could be interpreted as temporal variations in the density of the corotating plasma forced by geophysical activity on Io (Russell 2005). Europa has the property of a conducting sphere (Russell 2005) with a radius close to the solid surface radius. Given the low density of the kinetically collision free atmosphere derived here, the physical properties of the conducting sphere should be reevaluated.

The observational evidence in the region of the Galilean satellites shows consistency in magnitudes. Sittler \& Strobel (1987) from Voyager measurements show plasma sheet electron densities decreasing outward from $5.9 R_{J}$ with a sharp drop at $7.2 R_{J}$ coincident with a sharp rise in temperature, an indicator of a distinct decrease in mass loading. The evidence in the present work is consistent with this structure in showing a plasma at Europa that has converted the ion population to higher charge species at rates faster than dielectronic recombination and charge capture on neutral gas (Table 2). At $9.4 R_{J}$ during Voyager encounter the electron density was $\sim 40 \mathrm{~cm}^{-3}$, and $T_{e} \sim 250,000 \mathrm{~K}$.

The Roth et al. (2014) HST observation in 2012 December is unique in two respects: (1) two plumes are identified in the south polar region, giving emission in $\mathrm{H} \mathrm{Ly} \alpha$ and the O I $1304 \AA$ multiplet, indicators of dissociative excitation of $\mathrm{H}_{2} \mathrm{O}$. (2) The emission from the disk does not have verified $\mathrm{H} \mathrm{Ly} \alpha$ emission, but shows atomic oxygen emission at a mean brightness similar to all other reported observations (Table 3). Roth et al. (2014) report the emissions against the disk as auroral phenomena with microscale variability, but with brightness averaged over the disk at the same level as the other reported observations. Variability is certainly present in the system involving Europa, but the present research indicates it is a magnetospheric phenomenon rather than geophysical instability. The Io plasma torus is well known to be variable, with significant changes reported on timescales of months to years. As a primary forcing function on the system, Io will impose variability on the Europa environment (Russell 2005) and the energy for maintaining the plasma system through system rotation is obtained mainly from the sulfur ions derived from Io (see Ip et al. 1998). An event on Io propagates to the Europa orbit, but is filtered by the long physical chemistry and diffusion time constants. The general conclusion is that Europa does not show evidence of internal geophysical activity. The Saur et al. (1998) model for Europa has mass loading at a rate similar to that at Io. The mass loading rate at the Io torus is 
$\sim 7 \times 10^{27}$ atoms s $^{-1}$ (Shemansky 1988; Smyth \& Marconi 2006). The Saur et al. (1998) rate at Europa, $\sim 2 \times 10^{27}$ atoms $\mathrm{s}^{-1}$, is not plausible given the known conditions.

The calculations of O IV exchange sputtering (Table 4) indicate that this process, which diffuses dissociated atomic species into the vacuum, could be a significant contributor (along with the higher charged heavy heterogeneous ions) to the maintenance of the tenuous atmosphere.

The work presented here provides approximate quantities relevant to the state of the Europa environment. Model calculations on the detailed level of the energy balanced plasma calculations such as Shemansky (1988) and Yoshii (2011) for the Io and Enceladus environments will provide further insight on system forcing and the formation of the Europa atmosphere. Geophysical activity is sensitively monitored by atmospheric observation of Europa because the atmosphere is a hard vacuum and any minor event constitutes a large impact on the volume. Significant advances in understanding Europa's role in the system would be obtained, given the nature of the observations shown here, by high spatial resolution magnetospheric and atmospheric spectrally resolved photon emission measurements, particularly in the EUV.

The authors thank Professor Wing-Huen Ip for critical comments on the Galileo mission results at Jupiter. D.E.S., X.L., J.Y. and were partially supported by the Cassini UVIS program through contract to SET. Y.L.Y. was supported in part by the Cassini UVIS program via NASA grant JPL.1459109 to the California Institute of Technology. C.J.H. and A.R.H. were partially supported by the Cassini UVIS program through contract to PSI. L.W.E. was partially supported by the Cassini UVIS contract to LASP.

\section{REFERENCES}

Aumayr, F., \& Winter, H. 2004, RSPTA, 362, 77

Bagenal, F. 1994, JGR, 99, 11043

Cassidy, T. A., Paranicas, C. P., Shirley, J. H., et al. 2013, P\&SS, 7, 64

Cooper, J. F., Johnson, R. E., Mauk, B. H., Garrett, J. W., \& Gehrels, N. 2001, Icar, 149, 133
Esposito, L. E., Barth, C. A., Colwell, J. E., et al. 2004, SSRv, 115, 299

Hall, D. T., Feldman, P. D., McGrath, M. A., \& Strobel, D. F. 1998, ApJ, 499,475

Hall, D. T., Strobel, D. F., Feldman, P. D., McGrath, M. A., \& Weaver, H. A. 1995, Natur, 373, 677

Hansen, C. J., Shemansky, D. E., \& Hendrix, A. R. 2005, Icar, 176, 305

Hunten, D. M. 1995, Natur, 373, 654

Intriligator, D. S., \& Miller, W. D. 1982, JGR, 87, 8081

Ip, W.-H. 1996, Icar, 120, 317

Ip, W.-H., Williams, D. J., McEntire, R. W., \& Mauk, B. H. 1997, GeoRL, 24,2631

Ip, W.-H., Williams, D. J., McEntire, R. W., \& Mauk, B. H. 1998, GeoRL, 35,829

Itikawa, Y. 2003, JPCRD, 38, 1

Johnson, R. E., Boring, J. W., Reimann, C. T., et al. 1983, GeoRL, 10, 892

Killen, R., Shemansky, D., \& Mouawad, N. 2009, ApJS, 181, 351

Kliore, A. J., Hinson, D. P., Flasar, F. M., Nagy, A. F., \& Cravens, T. E. 1997, Sci, 277,355

Lagg, A., Krupp, N., \& Woch, J. 2003, GeoRL, 30, 1556

Liang, M. C., Lane, B. F., Pappalardo, R. T., Allen, M., \& Yung, Y. L. 2005, JGRE, 110, E02003

Lisse, C. M., Christian, D. J., Dennerl, K., et al. 2001, Sci, 292, 1343

Liu, X., Shemansky, D. E., Hallett, J. T., \& Weaver, H. A. 2007, ApJS, 169,458

Mauk, B. H., Mitchell, D. G., Krimigis, S. M., Roelof, E. C., \& Paranicas, C. P. 2003, Natur, 421, 920

Morgan, H. D., \& Mentall, J. E. 1974, JChPh, 60, 4734

Paonessa, M. 1982, JGR, 90, 521

Roth, L., Saur, J., Retherford, K. D., et al. 2014, Sci, 343, 171

Russell, C. T. 2005, P\&SS, 53, 473

Saur, J., Strobel, D. F., \& Neubauer, F. M. 1998, JGR, 103, 19947

Shemansky, D. E. 1980, ApJ, 242, 1266

Shemansky, D. E. 1988, JGR, 93, 1773

Shemansky, D. E. 2003, in AIP Conf. Proc. 663, 32rd Int. Symp., Rarefied Gas Dynamics, ed. A. D. Ketsdever \& E. P. Muntz (chapter 9; Melville, NY: AIP), 687

Shemansky, D. E., \& Smith, G. R. 1981, JGR, 86, 9179

Sittler, E. C., Jr., Cooper, J. F., Hartle, R. E., et al. 2013, P\&SS, 88, 26

Sittler, E. J., Jr., \& Strobel, D. F. 1987, JGR, 92, 5741

Smyth, W. H., \& Marconi, M. L. 2006, Icar, 181, 510

Smyth, W. H., \& Shemansky, D. E. 2005, ApJ, 271, 865 (21983)

Steffl, A. J., Bagenal, F., \& Stewart, A. I. F. 2004a, Icarus, 172, 91

Steffl, A. J., Stewart, A. I. F., \& Bagenal, F. 2004b, Icarus, 172, 78

Teolis, B. D., Vidal, R. A., Shi, J., \& Baragiola, R. A. 2005, PhRvB, 72,245422

Townsend, P. D., Kelley, J. C., \& Hartley, N. E. W. 1976, Ion Implantation, Sputtering, and Their Applications (New York: Academic)

Yoshii, J. 2011, PhD Disseration, Univ. Southern California 